\title{
MicroRNA-301 Mediates Proliferation and Invasion in Human Breast Cancer
}

\author{
Wei Shi ${ }^{1}$, Kate Gerster ${ }^{1}$, Nehad M. Alajez ${ }^{1,6}$, Jasmine Tsang ${ }^{1}$, Levi Waldron ${ }^{1}$, Melania Pintilie ${ }^{2}$, Angela B. Hui ${ }^{1}$, \\ Jenna Sykes ${ }^{2}$, Christine P'ng ${ }^{4}$, Naomi Miller ${ }^{5}$, David McCready ${ }^{3}$, Anthony Fyles ${ }^{4}$, and Fei-Fei Liu ${ }^{1,4}$
}

\section{Abstract}

Several microRNAs have been implicated in human breast cancer but none to date have been validated or utilized consistently in clinical management. MicroRNA-301 (miR-301) overexpression has been implicated as a negative prognostic indicator in lymph node negative (LNN) invasive ductal breast cancer, but its potential functional impact has not been determined. Here we report that in breast cancer cells, miR-301 attenuation decreased cell proliferation, clonogenicity, migration, invasion, tamoxifen resistance, tumor growth, and microvessel density, establishing an important oncogenic role for this gene. Algorithm-based and experimental strategies identified FOXF2, BBC3, PTEN, and COL2A1 as candidate miR-301 targets, all of which were verified as direct targets through luciferase reporter assays. We noted that miR-301 is located in an intron of the SKA2 gene which is responsible for kinetochore assembly, and both genes were found to be coexpressed in primary breast cancer samples. In summary, our findings define miR-301 as a crucial oncogene in human breast cancer that acts through multiple pathways and mechanisms to promote nodal or distant relapses. Cancer Res; 71(8); 2926-37. (C)2011 AACR.

\section{Introduction}

Since the initial discovery of microRNAs (miRNA) in 1993 (1), they have been shown to influence multiple cellular processes (2), and in particular, have been shown to play important roles in cancer development and progression $(3,4)$. The majority of miRNAs map to fragile genomic sites, and aberrant patterns of miRNA expression have been observed in multiple cancer types, including leukemias and lymphomas (5), colon (6), hepatocellular (7), head and neck (8), and breast cancers (2, 9-11). Functionally, aberrant miRNA expression can affect cell proliferation (12), apoptosis (4), chemo- and radiation-sensitivity (13), development of metastases (14), epithelial-mesenchymal transition (5), and could even potentially define the cancer stem cell phenotype (5).

Breast cancer is unfortunately the most commonly diagnosed cancer in women, and the second leading cause of cancer deaths in the developed world (15). The majority of

Authors' Affiliations: ${ }^{1}$ Department of Medical Biophysics, Ontario Cancer Institute; ${ }^{2}$ Biostatistics Group at Ontario Cancer Institute, ${ }^{3}$ Division of Surgical Oncology, and ${ }^{4}$ Department of Radiation Oncology, Princess Margaret Hospital, ${ }^{5}$ Department of Pathology, University Health Network, University of Toronto, Toronto, Canada; and ${ }^{6}$ Stem Cell Unit, Department of Anatomy, King Saud University, Riyadh, Saudi Arabia

Note: Supplementary data for this article are available at Cancer Research Online (http://cancerres.aacrjournals.org/).

Corresponding Author: Fei-Fei Liu, Department of Radiation Oncology, Princess Margaret Hospital/Ontario Cancer Institute, 610 University Avenue, Toronto, Ontario, Canada M5G 2M9. Phone: 416-946-2123; Fax: 416-946-4586; E-mail: Fei-Fei.Liu@rmp.uhn.on.ca

doi: 10.1158/0008-5472.CAN-10-3369

(C)2011 American Association for Cancer Research. women presenting with lymph node negative (LNN) disease fare well (16); however, the development of metastatic relapse is almost always incurable (17). Several studies have described putative breast cancer-specific miRNA signatures $(2,9,10,18)$; others have correlated miRNA expression with bio-pathologic features, including estrogen and progesterone receptor status (10). None to date, however, has been consistently validated, or utilized in clinical management.

In an effort to contribute to the understanding of miRNAs in human breast cancer, we had previously conducted a global miRNA profiling (11), wherein 4 miRNAs were identified to dichotomize relapse risk; 1 of which was hsa-miR301 , associated in particular with nodal and distant relapses. MicroRNA-301 (miR-301) has been previously noted to be deregulated in pancreatic (19), hepatocellular (20), and small cell lung cancers (21). It is located in the intron of SKA2 (spindle and kinetochore associated complex subunit 2), and was shown to regulate the expression of miR-301 in a lung cancer cell line (22), but little else is known about miR-301. In this study, we report that miR-301 promotes breast cancer proliferation, invasion, and tumor growth, mediated at least by FoxF2, BBC3, and PTEN. Furthermore, miR-301 likely cooperates with its host gene SKA2, contributing to the aggressive breast cancer phenotype with promotion of distant disease.

\section{Materials and Methods}

\section{Patients}

Seventy-one formalin-fixed, paraffin-embedded (FFPE) blocks of the 769 participants in a phase III clinical trial 


\begin{tabular}{ll} 
Table 1. Clinical characteristics of patients \\
Characteristic & Number (\%) \\
\hline Age, y & \\
$\leq 65$ & $39(55)$ \\
$>65$ & $32(45)$ \\
Treatment assignment & \\
Tamoxifen & $33(47)$ \\
Tamoxifen + radiotherapy & $38(53)$ \\
Tumor size, cm & \\
$\leq 2$ & $53(75)$ \\
$>2$ & $18(25)$ \\
Tumor overall NHS grade & \\
1 & $8(11)$ \\
2 & $33(47)$ \\
3 & $30(42)$ \\
ER & $60(85)$ \\
Positive $(>10 \%)$ & $11(15)$ \\
Negative $(\leq 10 \%)$ & \\
PR & $41(58)$ \\
Positive $(>10 \%)$ & $22(31)$ \\
Negative $(\leq 10 \%)$ & $8(11)$ \\
Unknown & \\
Her-2/neu & $5(7)$ \\
Positive & \\
Negative & \\
Equivocal &
\end{tabular}

Abbreviation: NHS, Nottingham histologic score; ER, estrogen receptor.

aUniform, intense complete cytoplasmic membrane staining of more than $30 \%$ invasive tumor cells.

${ }^{b}$ No cytoplasmic membrane staining or weak incomplete membrane staining in any proportion of invasive tumor cells. ${ }^{\mathrm{C} C o m p l e t e}$ circumferential membrane staining that is either non uniform or weak in $10 \%$ or more of invasive tumor cells or intense complete cytoplasmic membrane staining in 30\% or less of invasive tumor cells.

comparing Tamoxifen versus tamoxifen plus breast radiotherapy for LNN breast cancer (16) served as the cohort for miRNA profiling evaluation. The current study utilized a case control design. Case patients $(n=33)$ were identified as any individual who experienced local, lymph node, or distant metastasis within 10 years. Controls $(n=38)$ were identified as patients who had never experienced any relapse or death from cancer with $>10$ years' follow-up. The clinical characteristics of these 71 patients are described in Table 1. Five reduction mammoplasty specimens served as normal comparators. All studies have received Research Ethics Board approval from the University Health Network.

\section{Cell lines and transfections}

Primary human breast cancer cell lines MCF-7, MDA-MB231, MDA-MB-468, and T47D were obtained from American Type Culture Collection (2005-2007), and freshly recovered from liquid nitrogen ( $<6$ months). They were cultured in RPMI 1640 supplemented with 10\% FBS. MCF-10A cells were obtained from Dr. Linda Penn (Ontario Cancer Institute), and grown in DMEM/HAM F12 supplemented with 5\% horse serum, insulin, hEGF, hydrocortisone (Clonetics), and cholera toxin (Sigma). All cells have been authenticated using Short Tandem Repeat analyses, and are regularly examined using light microscopy. All cells are tested and determined to be free from Mycoplasma contamination every month.

The cells were transfected using the LipofectAMINE 2000 (Invitrogen) reverse transfection protocol, according to the manufacturer's instructions. AntagomiR-301, anti-miR negative control 1 (NC; Ambion), siRNA-SKA2, and scrambled siRNA ( $\mathrm{siC}$ ) were all transfected at a final concentration of $40 \mathrm{nmol} / \mathrm{L}$.

\section{Quantitative real-time PCR analysis of miRNAs and mRNAs}

The expression of hsa-miR-301 was measured using the standard Taqman MicroRNA Assay (Applied Biosystems) as previously reported (11). The primers used for mRNA expression are listed in Supplementary Table S1. RNA was isolated using the Norgen kit (Norgen Biotek Corporation).

\section{Cell proliferation and colony-forming assays}

Cytopathic effects of depleting miR-301 were evaluated in MCF-7 and T47D cells using the MTS assay (Promega), which is a colorimetric method for determining the viable cells in proliferation and cytotoxicity. For colony formation assays, MCF-7 and T47D cells were seeded onto plates 48 hours after transfection, incubated for 2 weeks, then fixed and stained, followed by colony counting.

\section{Cell migration and invasion assays}

For scratch migration assay, antagomiR-301 or NC-transfected cells were scratched using a standard 200 AL tip. Serial photographs were obtained at different time point using an Axiovert $100 \mathrm{M}$ phase contrast microscope (Carl Zeiss, Inc.). Invasion of MDA-MB-231 cells was assessed using the Matrigel Invasion Chamber (BD Biosciences), with conditions detailed in the Supplementary Materials and Methods.

\section{Target identification by mRNA profiling}

MCF-7 and MB-231 cells were transfected with either antagomiR-301 or NC. RNA was isolated 24 and 72 hours later, and gene expression was analyzed using the Whole Human Genome $4 \times 44 \mathrm{~K}$ Array (Agilent). Genes with greater than 2 -fold expression change relative to $\mathrm{NC}$ were considered as potential targets of miR-301, hence selected for further investigation.

\section{Western blotting}

MDA-MB-231 cells were transfected with either antagomiR301 or NC, then harvested on ice after 72 hours. Proteins were probed with mouse anti-FoxF2 (Abnova), rabbit anti-PTEN, pAkt (S473), total Akt (Cell Signaling Technology), rabbit antiPUMA BBC3 (Novus Biologicals LLC), or anti-GAPDH (Abcam). SKA2 protein was detected using a rabbit antiSKA2 antibody (ProSci Inc.). 


\section{Luciferase assay}

The 3 '-UTRs of FoxF2, PTEN, BBC3 and Col2A1 were amplified by PCR and cloned downstream of the luciferase gene in a pMIRREPORT luciferase vector (Ambion). A mutant sequence was also cloned as a validation plasmid. Either pMirluciferase or pMir-luciferase gene specific vectors were cotransfected with antagomiR-301 or NC in MDA-MB-231 or MCF-7 cell lines. pRL-SV vector (Promega) containing Renilla luciferase was also transfected with each condition as a reference control. Firefly and Renilla luciferase activities were measured using the Dual-Luciferase Reporter Assay (Promega).

\section{In vivo experiments}

Six- to 8-week-old severe combined immunodeficient mice (SCID) CB.17 female mice were utilized for the xenograft experiments. MDA-MB-231 cells were transfected with antagomiR-301, NC or LipofectAMINE 2000. At 48 hours posttransfection, cells were harvested, $5 \times 10^{5}$ viable cells were diluted in $100 \mu \mathrm{L}$ culture medium, then mixed with $100 \mu \mathrm{L}$ of Matrigel (BD Biosciences). Cells were then injected subcutaneously into the flank of SCID mice. Tumor volume was measured twice weekly using a caliper, calculated as (tumor length $\times$ width $\left.^{2}\right) / 2$. MCF-7 xenograft studies were conducted as described, but with the addition of implanting a $17 \beta$-estradiol pellet subcutaneously 1 day before cell injection.

\section{Detection of miR-301 using in situ hybridization}

In situ hybridization (ISH) was performed using a catalyzed signal amplification method (GenPoint signal amplification system; DakoCytomation) using the $5^{\prime}$ biotin-labeled miR-301 miRCURYTM locked nucleic acid detection probe, or a scrambled negative control probe (50 nmol/L; Exiqon). Positive hybridization signals were visualized by adding the chromogenic substrate diaminobenzidine.

\section{Immunohistochemistry for SKA2, CD31, and Ki-67}

Expression of SKA2, CD31, and Ki-67 were evaluated on tumor sections using microwave antigen retrieval in combination with the Level-2 Ultra Streptavidin system. Rabbit polyclonal anti-human SKA2 antibody (1:200 dilution; PorSci Inc.), rat anti-mouse CD31 antibody (1:50 dilution; BD Pharmingen), and mouse anti-human Ki-67 antigen (1:100 dilution; DakoCytomation) were utilized.

\section{Statistical analysis}

Box plots were utilized to visually explore the relationship between miR-301 or SKA2 expression using a Mann-WhitneyWilcoxon test. All other data were expressed as the mean \pm standard error (SE); a value of $P<0.05$ was considered to be statistically significant.

\section{Results}

miR-301 was overexpressed in LNN breast cancer, strongly associated with tumor recurrence

Global miRNA profiling was conducted on 71 archival FFPE LNN invasive ductal breast cancer samples using the TaqMan
Low Density Array (Panel v1.0; Applied Biosystems). In comparison with 5 normal mammary epithelial tissues, 57 miRs were significantly deregulated with adjusted values of $P<0.05$, amongst which 54 were upregulated and 3 were downregulated (data not shown). Amongst the top deregulated miRNAs was miR-301, which was $\sim 6.7$-fold upregulated, compared to normal tissues (Supplementary Fig. S1A). Furthermore, patients with higher miR-301 expression level experienced a worse DFS survival compared to those with lower miR-301 expression (Supplementary Fig. S1B). Most interestingly, the relapses associated with miR-301 expression were nodal or distant metastases, not local recurrences (Fig. 1A and Supplementary Fig. S1C).

\section{AntagomiR-301 caused significant reduction in miR- 301 expression, leading to reduced viability and decreased clonogenicity}

The expression of miR-301 was evaluated in 4 human breast cancer cell lines: MCF-7, T47D, MDA-MB-231, and MDA-MB468 , compared to that of the normal human mammary epithelial cell line MCF-10A. As shown in Figure 1B, miR301 was overexpressed in all 4 cell lines, indicating that these models are reasonable models for the primary human cancer scenario. To assess the biological role of miR-301, antagomiR301 was utilized in MCF-7 and T47D cells to knockdown its expression, showing a more than 9-fold reduction as early as 24 hours posttransfection, persisting for 72 hours (Fig. 1C).

This reduction in miR-301 led to a decrease in viability in both MCF-7 and T47D cells by $40 \%$ and $50 \%$ respectively at 72 hours posttransfection (Fig. 1D), which in turn translated to reduced clonogenicity (Fig. 1E), more so in the MCF-7 than the T47D cells (50\% and $80 \%$, respectively), concordant with the MTS data (Fig. 1D).

\section{Reducing miR-301 expression can increase sensitivity to tamoxifen}

Given that all the participants in this clinical trial were treated with tamoxifen (16), and the association of miR-301 with breast cancer relapses, this raised the question as to whether miR-301 could modulate tamoxifen sensitivity. A dose-response curve was first established, demonstrating that MCF-7 cells (estrogen-receptor positive) were indeed sensitive to 4-hydroxy-tamoxifen, with an $\mathrm{IC}_{50}$ of $300 \mathrm{nmol} / \mathrm{L}$ at 48 hours (Supplementary Fig. S2A). When MCF-7 cells were cotreated with antagomiR-301 plus 4-hydroxy-tamoxifen (300 nmol/L), a time-dependent cytotoxicity was observed, down to $38 \%$ at 48 hours, compared to NC, antagomiR alone, or NC with tamoxifen (Fig. 1F). However, this observation only applied at the $\mathrm{IC}_{50}$ dose of 4-hydroxy-tamoxifen $(300 \mathrm{nmol} / \mathrm{L})$. A similar result was also observed in the T47D cells (Supplementary Fig. S2B), suggesting that miR-301 depletion could modulate tamoxifen sensitivity, although this appeared to be dependent on the dose of tamoxifen.

\footnotetext{
AntagomiR-301 regulated cell migration and invasion, in addition to suppressing tumor growth in vivo

Given the strong association between miR-301 overexpression with nodal or distant relapse, we asked whether miR-301
} 


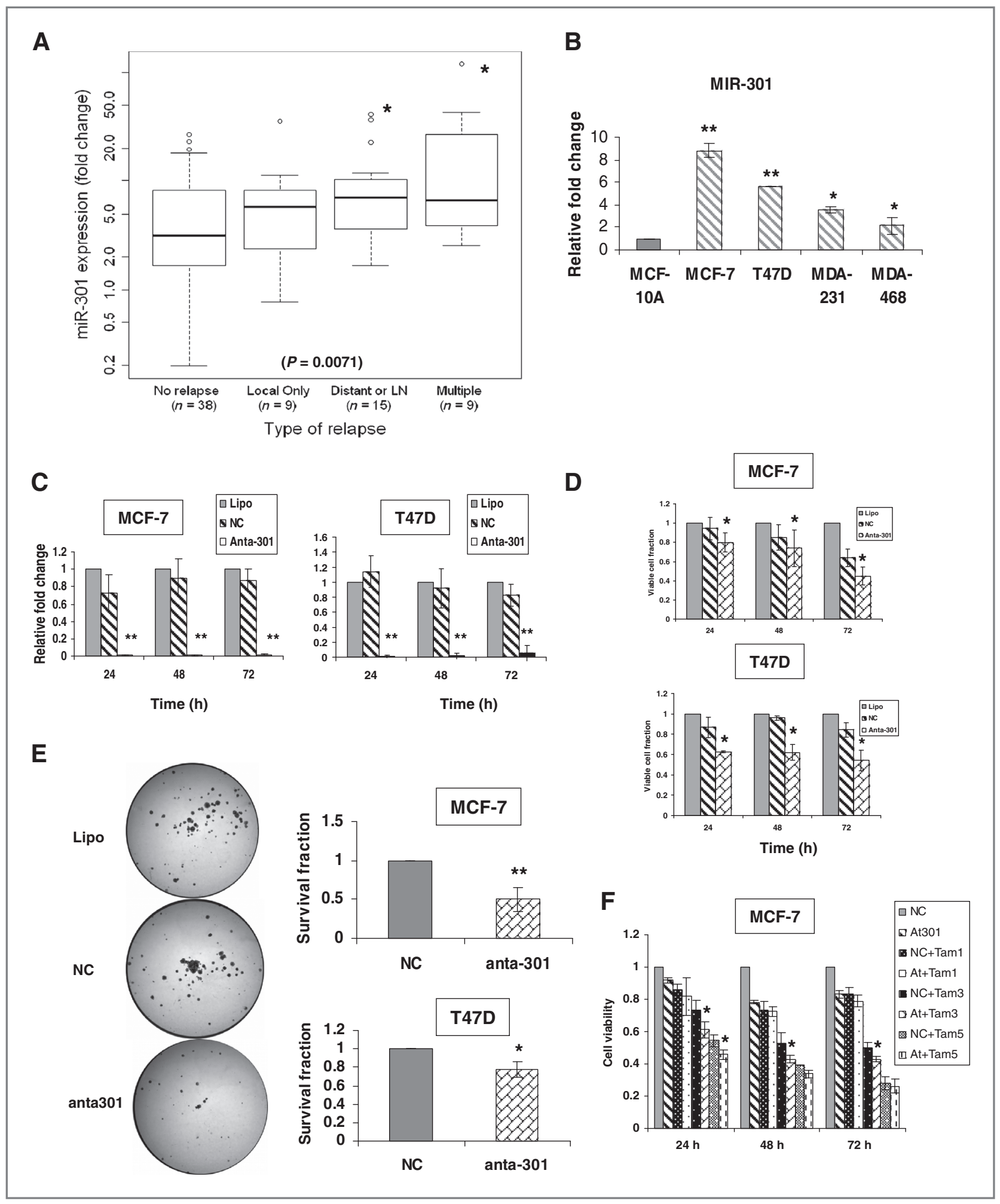

Figure 1. miR-301 overexpression in LNN breast cancer was strongly associated with tumor relapse. AntagomiR-301 caused significant reduction in miR-301 expression, leading to reduced viability, decreased clonogenicity, and increased sensitivity to tamoxifen. A, overexpression of miR-301 was strongly associated with distant/nodal ( $\left.{ }^{*}, P=0.03\right)$ or multiple $\left.{ }^{*}, P=0.02\right)$ relapses. The Mann-Whitney-Wilcoxon test was used to explore the relationship between miR-301 expressions with relapse type. B, miR-301 was overexpressed in all 4 human breast cancer cell lines. C-E, antagomiR-301 significantly reduced cell viability and decreased clonogenicity in both MCF-7 and T47D cells. F, depleting miR-301 increased tamoxifen sensitivity ( $300 \mathrm{nmol} / \mathrm{L}$ ) in MCF-7 cells. Each datum represents the mean fold change $\pm \mathrm{SE}$ from triplicates. AntagomiR versus $\mathrm{NC},{ }^{*}, P<0.05,{ }^{*}, P<0.001$. 
knockdown could affect cell migration or invasion. Indeed, using the scratch assay, antagomiR-301 did reduce migration for both MCF-7 and MDA-MB-231 cells (Fig. 2A). Furthermore, miR-301 depletion reduced the invasive ability of the aggressive MDA-MB-231 cells to $44 \%$ versus $62 \%$ for NC cells $(P<$ 0.05; Fig. 2B).

The reduced clonogenicity in vitro translated to the in vivo model wherein antagomiR-301-treated MDA-MB-231 or MCF-7 cells showed significantly delayed tumor growth compared to mice injected with either NC- or Lipofectamine-treated cells $(P=0.04$ and $P=0.05$, respectively; Fig. 2C). To acquire further insights into the mediators of miR-301 suppression leading to reduced tumor growth, tumors from the NC- and antagomiR-301-treated mice were collected 45 days after implantation. Figure 2D showed reduced tumor microvessel density, observed via CD31 immunoexpression in the antagomiR-301-treated MDAMB-231 tumor compared to a control tumor. Ki-67 expression was only slightly reduced (data not shown), indicating that miR-301 affected tumor angiogenesis.

\section{miR-301 directly targets FoxF2, PTEN, BBC3iso-2, and Col2A1 in breast cancer cells}

These phenotypic data thus far indicate that miR-301 overexpression causes a myriad of effects including breast cancer cell proliferation, migration, invasion, along with tumor growth and angiogenesis. To identify its potential mRNA targets which could mediate these changes, a tripronged approach was undertaken $(23,24)$; details provided in Supplementary Figure S2C. Fourteen overlapped candidate targets were thus identified (FoxF2, BBC3, PTEN, CoL2A1, ITGB8, IL18, MMP13, Bcl2L14, ARRP1, E2F2, RAG1, IGFBP2, IGFBP5, and SMOC2). Individual quantitative real-time PCR (qRT-PCR) assays showed that the basal expression of 7 of these candidate transcripts were underexpressed in the majority of the 4 human breast cancer cell lines (FoxF2, PTEN, BBC3, Col2A1, Il18, ITGB8, and MMP13), which were thus selected for further evaluation (Supplementary Fig. S3). Knocking down miR-301 induced the expression modestly in 5 of 7 mRNA transcript levels for both MCF-7 and MDA-MB-231 cells, by only 1.1 to 2 -fold, at either 48 or 72 hours posttransfection (Fig. 3A). Western blotting confirmed increased protein expression for FoxF2, PTEN, BBC3 with reduced expression of p-Akt, compared to NC (Fig. 3B).

The direct interaction of miR-301 with the top 4 candidate mRNA transcripts: FoxF2, PTEN, BBC3, Col2A1 was clearly shown by the induction of their respective luciferase expression by 1.2 to 2 -fold when MDA-MB-231 cells were cotransfected with antagomiR-301 and the luciferase reporter plasmid carrying the relevant wild-type $3^{\prime}$-UTR sequences, which was completely abrogated by the mutant $3^{\prime}$-UTR sequences (Fig. 3C). Almost identical data were observed for the MCF-7 cells (Supplementary Fig. S4A), thereby corroborating that these 4 mRNA transcripts were indeed bona fide targets for miR-301.

In order to identify some of the further downstream effectors of the observed cellular phenotypic changes, Wnt5a, a target of FoxF2 was evaluated post-miR-301 depletion. The overexpression of Wnt5a has been correlated with increased cell motility and invasion in melanoma and breast cancer cells (25). In 3 of 4 tested breast cancer cell lines, the basal expression of Wnt-5a was slightly increased (Supplementary Fig. S4B). Depletion of miR-301 in turn reduced Wnt5a expression (Fig. 3D), suggesting that miR-301 could be regulating Wnt5a via FoxF2, at least in the MCF-7 and MDA-MB-231 cells.

Given the reduced microvessel density observed in the MDA-MB-231 tumors when miR-301 was depleted, and VEGF being a known downstream effecter of PTEN-Akt, we asked whether VEGF levels would be differentially expressed in our primary human breast cancer samples. Indeed, the median VEGF transcript expression level analyzed in the remaining samples with sufficient RNA was 7.7-fold higher than normal tissues, compared to 15.4-fold between the nonrelapsed versus relapsed breast cancer patient samples (Fig. 3E), suggesting that VEGF could indeed be a downstream effecter of breast cancer relapse.

\section{SKA2 is the host gene of miR-301}

It is well known that more than $50 \%$ of mammalian miRNA genes are located within introns of protein-coding or noncoding units (26), wherein miRNAs could be transcribed in parallel with their host genes. Interrogation of the Sanger miRNA database revealed that miR-301 overlaps with the first intron of the SKA2 gene, located on $17 \mathrm{q} 22$ (Fig. 4A). SKA2 is a recently described member of the SKA complex, which is essential for proper chromosomal segregation (27). SKA2 has been shown to be overexpressed in human lung and breast cancer tissues, perhaps mediating cancer cell proliferation (28). Hence, the expression of SKA2 was investigated in the same LNN breast cancer specimens using qRT-PCR. Amongst the 64 tumors examined (with sufficient remaining RNA), 44 showed upregulation of SKA2, with 37 (84\%) exhibiting overexpression of both SKA2 and miR-301 (Supplementary Fig. S5A). Interestingly, SKA2 overexpression was also significantly associated with only a higher risk of nodal or distant relapse ( $P=0.03$; Fig. 4B). Similarly, SKA2 overexpression was also observed in all 4 human breast cancer cell lines (Fig. 4C), which when knocked down using siRNA, led to a significant reduction in cell viability down to $50 \%$ in the MCF-7, or $70 \%$ in the MDA-MB-231 cells, in a time-dependent manner up to 72 hours posttransfection (Fig. 4E). Reduced mRNA and protein levels of SKA2 after siSKA2 were corroborated using qRT-PCR and Western blotting, respectively (Fig. 4D). These data indicated that SKA2 could also independently influence tumor cell proliferation in vitro.

\section{Colocalization of miR-301 and SKA2 in tumor cells}

Finally, to further ascertain that miR-301 and SKA2 were indeed coexpressed in primary human breast cancer, miR301 was visualized using in situ hybridization, and SKA2 via immunohistochemistry. As shown in Figure 5A and B, intense miR-301 signals were observed in the cytoplasm of tumor cells, not observed with a scrambled control probe (Supplementary Fig. S5B). In a serial section of the 


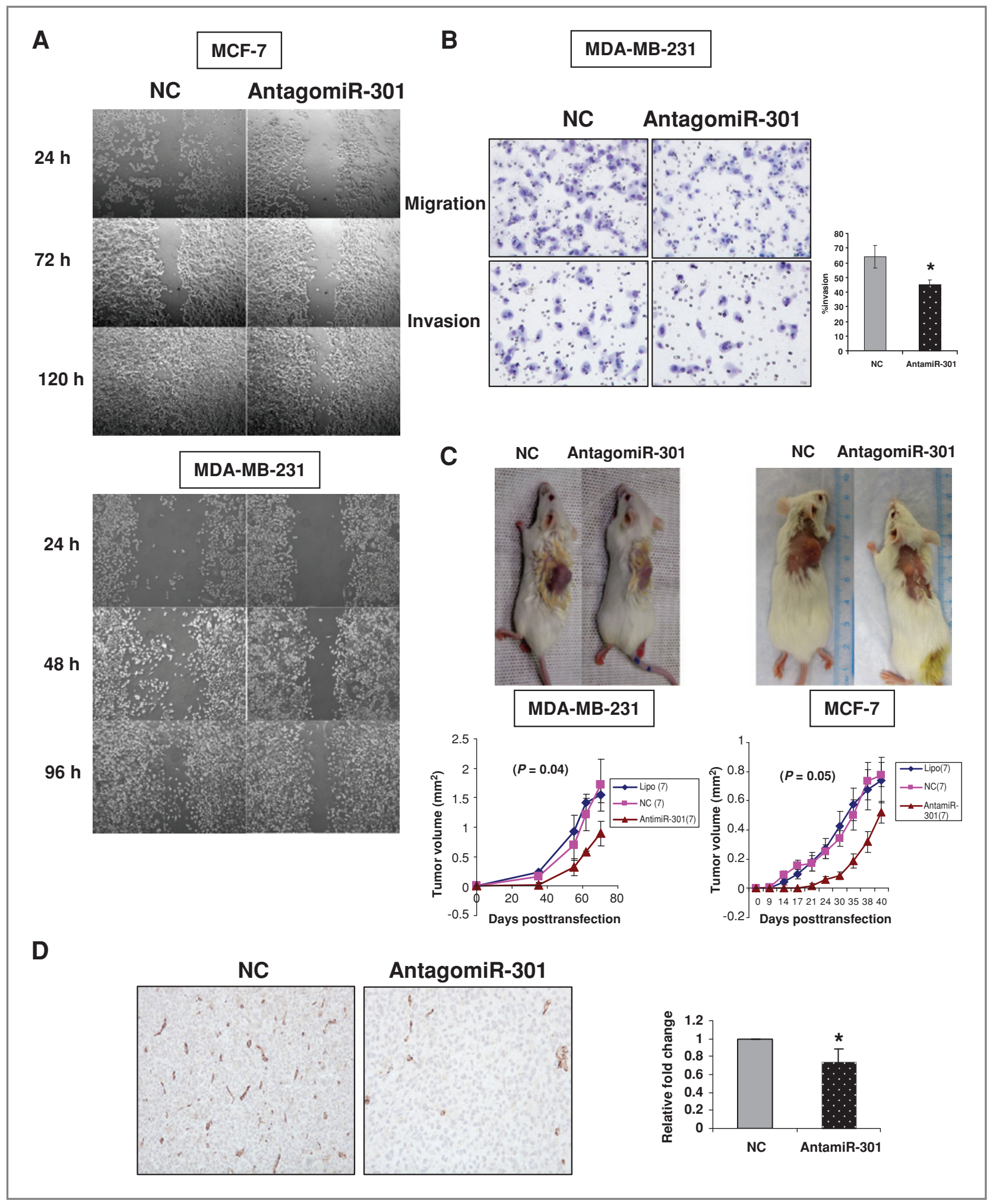

Figure 2. Knockdown of miR-301 significantly reduced cell migration and invasion, in addition to suppressing tumor growth, and microvessel density. A, the scratch migration assay. Serial photographs were obtained at indicated times posttransfection in MCF-7 and MDA-MB-231 cells. B, the migration and invasion rates were determined by counting the number of cells which have migrated onto collagen IV-coated plates (upper), or invaded through Matrigel (bottom). C, tumor formation in SCID mice after subcutaneous injection of transfected-MDA-MB-231 or MCF-7 cells. The indicated tumor volumes represent the mean $\pm \mathrm{SE}$. D, representative photomicrograph of CD31 immunostaining in MDA-MB-231 xenograft tumors; quantitation of the number of microvessels in 10 representative hpf's; the datum represents the mean $\pm \mathrm{SE}$ from 2 independently treated tumors $\left(^{*}, P<0.05\right)$. 


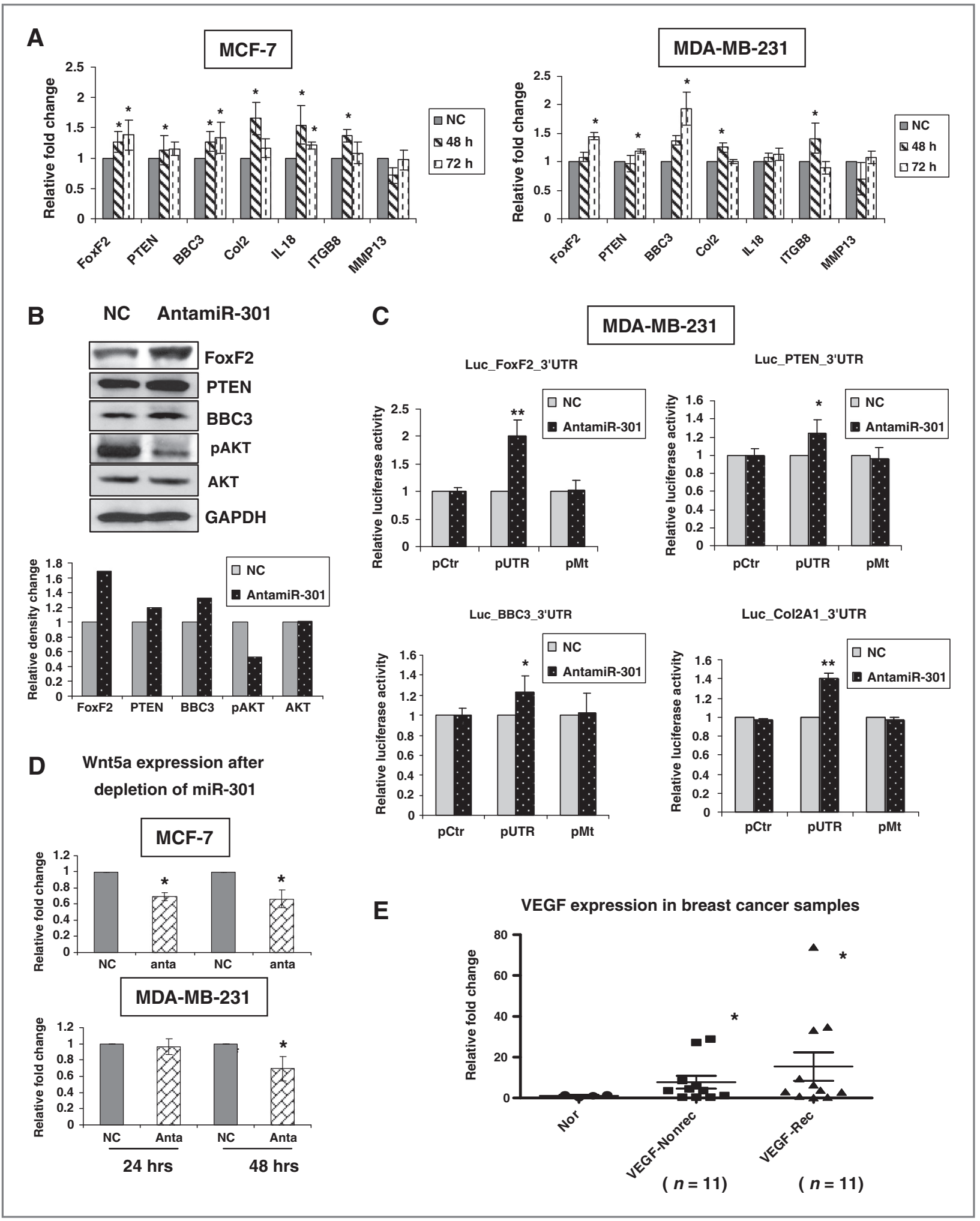

Figure 3. miR-301 target identification and validation. A tripronged approach identified 7 downregulated genes as potential targets for miR-301. A, qRT-PCR showed upregulation in 6 and 5 of the tested 7 genes after miR-301 knockdown in the MCF-7 and MDA-MB-231 cells, respectively. B, Western blotting for FoxF2, PTEN, BBC3, p-Akt, and total Akt at 72 hours posttransfection of antagomiR-301 in MDA-MB-231 cells. C, luciferase reporter assays confirmed that FoxF2, PTEN, BBC3, and Col2A1 were direct targets of miR-301 in the MDA-MB-231 cells, with luciferase activity ranging from 1.2 to 2-fold induction. D, Wnt5a expression was reduced after miR-301 knockdown for both MCF-7 and MDA-MB-231 cells. Each datum represents the mean fold change in expression from 3 independent experiments \pm SE. E, pretreatment expression VEGF in nonrecurrent $(n=11)$ versus recurrent $(n=11)$ primary human breast cancer samples compared with 4 normal mammoplasty specimens (Nor). ${ }^{*}, P<0.05$. 


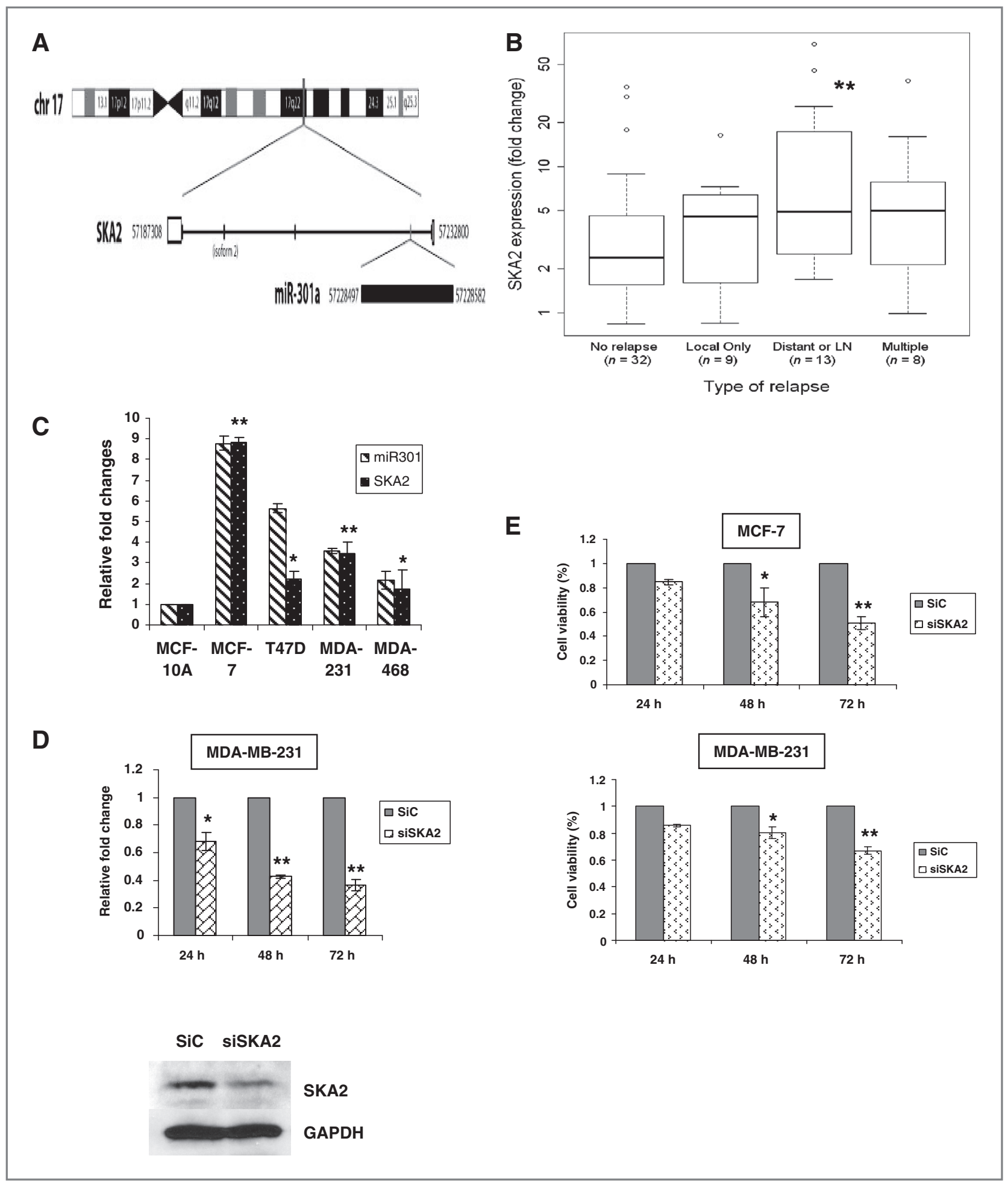

Figure 4. SKA2 is the host gene for miR-301. A, a schema demonstrating the location of miR-301 on chromosome 17q22, within the intron of SKA2. $\mathrm{B}$, box plot demonstrating a correlation between SKA2 expression with distant or nodal relapses. ${ }^{*}, P<0.04$. C, qRT-PCR measurements of SKA2 overexpression in all 4 human breast cancer cells, relative to that of MCF-10A. D, reduced mRNA and protein levels of SKA2 after siSKA2 in MDA-MB-231 cells showed using qRT-PCR and Western blotting, respectively. E, SKA2 knockdown significantly reduced cell viability in both cell lines. Each datum represents the mean fold change from 3 independent experiments \pm SE. ${ }^{*}, P<0.05$, ${ }^{\star *}, P<0.001$. 


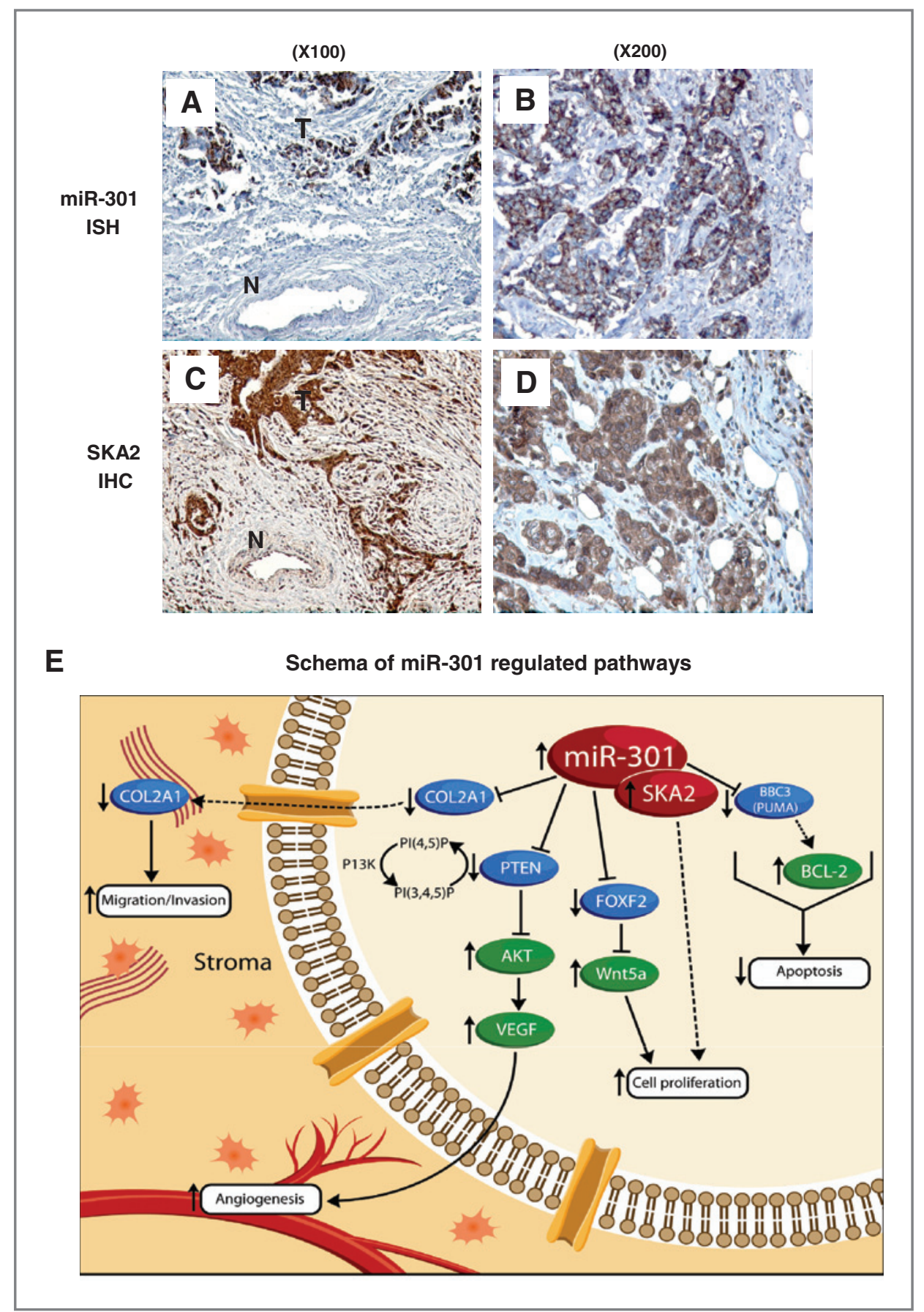

Figure 5. Colocalization of miR301 and SKA2 in a primary breast cancer specimen. In situ

hybridization (ISH) demonstrating granular brown cytoplasmic expression of miR-301 in tumor (T), but not in the normal tissues (N). A, 100x; B, 200x

magnifications. In an adjacent serial 4- $\mu \mathrm{m}$ section,

immunohistochemistry showed significant cytoplasmic

expression of SKA2 also in tumor (T), but not in normal tissues. C, $100 \times ; \mathrm{D}, 200 \times$ magnifications. E, a proposed model wherein miR301 overexpression in human breast cancer can downregulate several mRNA targets, including Col2A1, PTEN, FoxF2, and BBC3. In turn, downregulation of Col2A1 can lead to increased migration and invasion. Similarly, PTEN downregulation will lead to Akt activation, which can promote VEGF expression, with increased tumor angiogenesis.

Downregulation of FoxF2 can increase expression of Wnt5a, which can promote cell proliferation. In parallel, SKA2 is also coexpressed with miR-301, which can also potentially increase proliferation.

same patient's tumor, SKA2 immunoexpression was also observed in the tumor cytoplasm (Fig. 5C and D); again not in the negative control (Supplementary Fig. S5C). Note that neither miR-301 nor SKA2 was observed in the surrounding normal tissues. This therefore provided the visual corroboration of the previously shown qRT-PCR data for the co-overexpression of both miR-301 and SKA2 in primary human breast cancer tissues (Supplementary Fig. S5A).

\section{Discussion}

Current clinical decisions on breast cancer management for LNN patients are based on parameters such as age, menopausal status, tumor size, and histology, which are suboptimal, and likely result in the overtreatment of many of our patients (29). Several molecular prognostic markers have been proposed (30); the most frequently utilized set in North America is Oncotype DX, a 21-gene assay applicable to LNN estrogen 
receptor-positive cancers (31). The true utility of these predictive signatures, however, are still under evaluation, underscoring the biological complexities of LNN disease; hence the importance of determining whether novel insights could be acquired through the lens of miRNAs.

The current study reported miR-301 as a novel oncogene in LNN breast cancer. miR-301 overexpression was associated with an increased risk of nodal or distant relapse, and mediated proliferation, migration, invasion, resistance to tamoxifen, and tumor formation with increased angiogenesis. These pleiotropic effects of miR-301 were directly mediated by several well-known oncogenic targets and pathways, including PTEN, FoxF2, BBC3, and Col2al. Furthermore, its coexpression with SKA2 likely cooperatively promotes breast cancer progression (Fig. 5E).

Dysregulation of the PTEN-PI3K/Akt signaling pathway is well characterized in its myriad of roles in causing proliferation, increasing angiogenesis, migration, and invasion, leading to metastases (32). In fact, we had previously reported dysregulation of the PTEN-PKB axis in primary human breast cancer samples (33). Although the mechanism of dysregulation was unclear, further examination of the 29 overlapping samples (from the previous and the current cohorts), showed that 14 samples overexpressed both miR-301 and PTEN; 5 overexpressed miR-301 with concomitantly low PTEN; and 10 were reversed with low miR-301 and high PTEN expression $(P=$ 0.07 ), suggesting that in this small cohort, there might indeed be a trend of a reciprocal relationship between miR-301 with PTEN. In the current study, PTEN appears to be a central target of miR-301 in mediating many of the observed phenotypic changes in the breast cancer cells consequent to its knock down. We observed increased proliferation, migration, and invasion in vitro (Figs. $1 \mathrm{D}$ and $\mathrm{E}$ and $2 \mathrm{~A}$ and $\mathrm{B}$ ), which can certainly all be attributed by the mitogenic signals of a downregulated PTEN cascade, leading to activated PI (3) K-Akt, with multiple further downstream signals involving matrix metalloproteinases, and integrins (34). Furthermore, we observed enhanced tumor formation, associated with increased microvessel density in vivo, corroborated by increased VEGF mRNA expression in the same primary breast cancer tissues, which in turn was associated with relapse (Fig. 3E); again, these phenotypes would be consistent with an activated Akt cascade (34).

FoxF2 is a member of the forkhead family of transcription factors (35), which regulate diverse cellular processes (36). FoxF2 has been recently described to be underexpressed in prostate cancer (37), and can downregulate Wnt5a (38). Our data show that FoxF2 is directly targeted by miR-301, and miR301 depletion reduced Wnt5a expression (Fig. 3). Wnt5a activates the noncanonical Wnt pathways, with distinct signaling and biological phenotypes depending on context, and availability of partner receptors (39). There is controversy regarding the role of Wnt5a in human malignancies, with reports of both tumor suppressive (40), and oncogenic effects (41). Certainly Wnt5a can mediate cell polarity, migration, and invasion through its interactions with the Frizzled receptors (39), which would be concordant with our observations via the miR-301/ FoxF2/Wnt5a axis (Fig. 5E), although clearly, the roles of FoxF2 and Wnt5a in human malignancies are just beginning to be understood, definitely warranting further examination.
In addition to elucidating the cancer relevant pathways downstream of miR-301 such as the aforementioned PTEN and FoxF2, we also noted that miR-301 was located within the first intron of SKA2 (42). Not only were both miR-301 and SKA2 co-overexpressed in our breast cancer samples (Fig. 5, Supplementary Fig. S5A), but SKA2 with miR-301 also associated with clinical outcome in that the worst disease-free survival was observed for the tumors which overexpressed both genes versus the best outcome when neither was overexpressed (Supplementary Fig. S6A). SKA2 forms at the kinetochoremicrotubule interface during mitosis $(27,43)$. Transient depletion of the SKA complex destabilizes the microtubulekinetochore attachment, resulting in metaphase arrest, while more stringent and prolonged SKA depletion causes chromosome congression defects with subsequent cell death (27). Very little is known about SKA2 in human cancers, but a recent study with the A549 cancer cell line described miR-301 to be indirectly upregulating SKA2, and its depletion reduced colony formation in agar (22). Their observations would be consistent with our observed phenotype wherein overexpression of SKA2 (with miR-301) appears to promote proliferation and tumor progression, although the precise mechanisms and pathways remain to be elucidated.

The 2 other direct targets of miR-301, BBC3, and Col2A1 were shown at the transcript and protein levels along with luciferase reporter data (Fig. 3A-C, Supplementary Figs. S3 and S4). We were unable to show a significant antiapoptotic process after increasing BBC3 levels in any of our tested breast cancer cells. Col2Al encodes the $\alpha$ chain of type II collagen (44), which is an important component of the extracellular matrix (45). It is certainly conceivable that the migration and invasion observed after miR-301 depletion (Fig. 2A and B) could be mediated through the downregulation of Col2Al.

Finally, given that all the participants in this randomized clinical trial received tamoxifen, and miR-301 overexpression was associated with relapse, we asked whether miR-301 depletion could affect tamoxifen sensitivity, and indeed showed a modest effect in both MCF-7 and T47D cells (Fig. 1F, Supplementary Fig. S2B). Despite decades of clinical experience with tamoxifen, the precise mechanisms of tamoxifen resistance remain largely unknown. Our data do not clearly show the pathways of resistance, but activation of Akt has certainly been implicated (46); hence it is plausible that the miR-301/PTEN/ Akt pathway could be influencing tamoxifen sensitivity, based on our cell line and clinical observations.

In conclusion, our experiments have shown a novel pleiotropic oncogenic role for miR-301 through regulation of key signaling pathways involving at least PTEN, FOXF2, and Col2A1, and working in cooperation with SKA2. The resulting phenotype of an upregulated miR-301 includes increased proliferation, migration, invasion, and tumor formation. Furthermore, miR-301 overexpression is a candidate prognostic marker for metastases in patients with LNN breast cancer, which should be validated in a larger patient cohort.

\section{Disclosure of Potential Conflicts of Interest}

None of the authors have any conflict of interest. 


\section{Grant Support}

This work has been supported by funds from the Canadian Breast Cancer Foundation, and in part from the Campbell Family Institute for Cancer Research, and the Ministry of Health and Long-Term Planning.

\section{References}

1. Lee RC, Feinbaum RL, Ambros V. The C. elegans heterochronic gene lin-4 encodes small RNAs with antisense complementarity to lin-14. Cell 1993;75:843-54.

2. Iorio MV, Ferracin M, Liu CG, Veronese A, Spizzo R, Sabbioni S, et al. MicroRNA gene expression deregulation in human breast cancer. Cancer Res 2005;65:7065-70.

3. Croce CM. Causes and consequences of microRNA dysregulation in cancer. Nat Rev Genet 2009;10:704-14.

4. Esquela-Kerscher A, Slack FJ. Oncomirs-microRNAs with a role in cancer. Nat Rev Cancer 2006;6:259-69.

5. Nicoloso MS, Spizzo R, Shimizu M, Rossi S, Calin GA. MicroRNAsthe micro steering wheel of tumour metastases. Nat Rev Cancer 2009;9:293-302.

6. Chen X, Guo X, Zhang H, Xiang Y, Chen J, Yin Y, et al. Role of miR-143 targeting KRAS in colorectal tumorigenesis. Oncogene 2009;28: 1385-92.

7. Gramantieri L, Ferracin M, Fornari F, Veronese A, Sabbioni S, Liu CG, et al. Cyclin G1 Is a target of miR-122a, a microRNA frequently downregulated in human hepatocellular carcinoma. Cancer Res 2007; 67:6092-9.

8. Hui AB, Lenarduzzi M, Krushel T, Waldron L, Pintilie M, Shi W, et al. Comprehensive microRNA profiling for head and neck squamous cell carcinomas. Clin Cancer Res 2010;16:1129-39.

9. Blenkiron C, Goldstein LD, Thorne NP, Someone E. MicroRNA expression profiling of human breast cancer identifies new markers of tumor subtype. Genome Biol 2007;8:R214.

10. Silveri L, Tilly G, Vilotte J-L, Le Provost F. MicroRNA involvement in mammary gland development and breast cancer. Reprod Nutr Dev 2006:5:549-56.

11. Hui AB, Shi W, Boutros PC, Miller N, Pintilie M, Fyles T, et al. Robust global micro-RNA profiling with formalin-fixed paraffin-embedded breast cancer tissues. Lab Invest 2009;89:597-606.

12. Hiyoshi $Y$, Kamohara $H$, Karashima $R$, Sato N, Imamura $Y$, Nagai $Y$, et al. MicroRNA-21 regulates the proliferation and invasion in esophageal squamous cell carcinoma. Clin Cancer Res 2009;15:191522.

13. Ma J, Dong C, Ji C. MicroRNA and drug resistance. Cancer Gene Ther 2010;17:523-31.

14. Ma L, Teruya-Feldstein J, Weinberg RA. Tumour invasion and metastasis initiated by microRNA-10b in breast cancer. Nature 2007;449: 682-9.

15. Smith RA, Cokkinides V, Brooks D, Saslow D, Brawley OW. Cancer screening in the United States, 2010: a review of current American Cancer Society guidelines and issues in cancer screening. CA Cancer J Clin 2010;60:99-119.

16. Fyles AW, McCready DR, Manchul LA, Trudeau ME, Merante P, Pintilie M, et al. Tamoxifen with or without breast irradiation in women 50 years of age or older with early breast cancer. $\mathrm{N}$ Engl $\mathrm{J}$ Med 2004;351:963-70.

17. Retsky MW, Demicheli R, Hrushesky WJ, Baum M, Gukas ID. Dormancy and surgery-driven escape from dormancy help explain some clinical features of breast cancer. APMIS 2008;116:730-41.

18. Tavazoie SF, Alarcon C, Oskarsson T, Padua D, Wang Q, Bos PD, et al. Endogeneous human microRNAs that suppress breast cancer metastasis. Nature 2007;451:147-52.

19. Lee EJ, Gusev Y, Jiang J, Nuovo GJ, Lerner MR, Frankel WL, et al. Expression profiling identifies microRNA signature in pancreatic cancer. Int J Cancer 2007;120:1046-54.

20. Jiang J, Gusev Y, Aderca I, Mettler TA, Nagorney DM, Brackett DJ, et al. Association of MicroRNA expression in hepatocellular carcinomas with hepatitis infection, cirrhosis, and patient survival. Clin Cancer Res 2008;14:419-27.
The costs of publication of this article were defrayed in part by the payment of page charges. This article must therefore be hereby marked advertisement in accordance with 18 U.S.C. Section 1734 solely to indicate this fact.

Received September 13, 2010; revised February 9, 2011; accepted February 22, 2011; published OnlineFirst March 10, 2011.

21. Miko E, Czimmerer Z, Csánky E, Boros G, Buslig J, Dezso B, et al. Differentially expressed microRNAs in small cell lung cancer. Exp Lung Res 2009;35:646-64.

22. Cao G, Huang B, Liu Z, Zhang J, Xu H, Xia W, et al. Intronic miR-301 feedback regulates its host gene, ska2, in A549 cells by targeting MEOX2 to affect ERK/CREB pathways. Biochem Biophys Res Commun 2010;396:978-82.

23. Alajez NM, Lenarduzzi M, Ito E, Hui AB, Shi W, Bruce J, et al. miR-218 suppresses nasopharyngeal cancer progression through downregulation of survivin and the SLIT2-ROBO1 pathway. Cancer Res. In press 2011

24. Wang Y, Klijn J, Zhang Y, Atkins D, Foekens J. Gene expression profiles and prognostic markers for primary breast cancer. Methods Mol Biol 2007;377:131-8.

25. Weeraratna AT, Jiang Y, Hostetter G, Rosenblatt K, Duray P, Bittner $M$, et al. Wnt5a signaling directly affects cell motility and invasion of metastatic melanoma. Cancer Cell 2002;1:279-88.

26. Rodriguez A, Griffiths-Jones S, Ashurst JL, Bradley A. Identification of mammalian microRNA host genes and transcription units. Genome Res 2004;14:1902-10.

27. Gaitanos TN, Santamaria A, Jeyaprakash AA, Wang B, Conti E, Nigg EA. Stable kinetochore-microtubule interactions depend on the Ska complex and its new component Ska3/C13Orf3. EMBO J 2009;28: 1442-52.

28. Rice L, Waters CE, Eccles J, Garside H, Sommer P, Kay P, et al. Identification and functional analysis of SKA2 interaction with the glucocorticoid receptor. J Endocrinol 2008;198:499-509.

29. Roukos DH, Murray S, Briasoulis E. Molecular genetic tools shape a roadmap towards a more accurate prognostic prediction and personalized management of cancer. Cancer Biol Ther 2007;6:30812.

30. Dunn L, Demichele A. Genomic predictors of outcome and treatment response in breast cancer. Mol Diagn Ther 2009;13:73-90.

31. Paik S, Shak S, Tang G, Kim C, Baker J, Cronin M, et al. A multigene assay to predict recurrence of tamoxifen-treated, node-negative breast cancer. N Engl J Med 2004;351:2817-26.

32. Altomare DA, Testa JR. Perturbations of the AKT signaling pathway in human cancer. Oncogene 2005;24:7455-64.

33. Shi W, Zhang X, Pintilie M, Ma N, Miller N, Banerjee D, et al. Dysregulated PTEN-PKB and negative receptor status in human breast cancer. Int J Cancer 2003;104:195-203.

34. Chin YR, Toker A. Function of Akt/PKB signaling to cell motility, invasion and the tumor stroma in cancer. Cell Signal 2009;21: 470-6.

35. Katoh M. Human FOX gene family (Review). Int J Oncol 2004;25:1495500.

36. Gerin I, Bommer GT, Lidell ME, Cederberg A, Enerback S, MacDougald OA. On the role of FOX transcription factors in adipocyte differentiation and insulin-stimulated glucose uptake. J Biol Chem 2009;284:10755-63.

37. Van Der Heul-Nieuwenhuijsen L, Dits N, Van ljcken W, de Lange D, Jenster G. The FOXF2 pathway in the human prostate stroma. Prostate 2009;69:1538-47.

38. Ormestad M, Astorga J, Landgren H, Wang T, Johansson BR, Miura N, et al. Foxf1 and Foxf2 control murine gut development by limiting mesenchymal Wnt signaling and promoting extracellular matrix production. Development 2006;133:833-43.

39. McDonald SL, Silver A. The opposing roles of Wnt-5a in cancer. Br J Cancer 2009;101:209-14

40. Jönsson M, Dejmek J, Bendahl PO, Andersson T. Loss of Wnt-5a protein is associated with early relapse in invasive ductal breast carcinomas. Cancer Res 2002;62:409-16. 
41. Fernandez-Cobo M, Zammarchi F, Mandeli J, Holland JF, Pogo BG. Expression of Wnt5A and Wnt10B in non-immortalized breast cancer cells. Oncol Rep 2007;17:903-7.

42. Griffiths-Jones S, Saini HK, van Dongen S, Enright AJ. miRBase: tools for microRNA genomics. Nucleic Acids Res 2008;36:D154-8.

43. Hanisch A, Silljé HH, Nigg EA. Timely anaphase onset requires a novel spindle and kinetochore complex comprising Ska1 and Ska2. EMBO J 2006;25:5504-15.
44. Kannu P, Bateman JF, Randle S, Cowie S, du Sart D, McGrath S, et al. Premature arthritis is a distinct type II collagen phenotype. Arthritis Rheum 2010;62:1421-30.

45. Yilmaz M, Christofori G. EMT, the cytoskeleton, and cancer cell invasion. Cancer Metastasis Rev 2009;28:15-33.

46. Clark AS, West K, Streicher S, Dennis PA. Constitutive and inducible Akt activity promotes resistance to chemotherapy, trastuzumab, or tamoxifen in breast cancer cells. Mol Cancer Ther 2002;1:707-17. 


\section{Cancer Research}

\section{MicroRNA-301 Mediates Proliferation and Invasion in Human Breast Cancer}

Wei Shi, Kate Gerster, Nehad M. Alajez, et al.

Cancer Res Published OnlineFirst March 10, 2011.

Updated version

Supplementary

Material
Access the most recent version of this article at: doi:10.1158/0008-5472.CAN-10-3369

Access the most recent supplemental material at: http://cancerres.aacrjournals.org/content/suppl/2011/03/10/0008-5472.CAN-10-3369.DC1

E-mail alerts Sign up to receive free email-alerts related to this article or journal.

Reprints and To order reprints of this article or to subscribe to the journal, contact the AACR Publications Subscriptions Department at pubs@aacr.org.

Permissions To request permission to re-use all or part of this article, contact the AACR Publications Department at permissions@aacr.org. 